\title{
Identificación de cuatro especies de Clupeiformes (Actinopterygii) mediante análisis de secuencias de ADN mitocondrial en zonas de explotación pesquera del Golfo de Nicoya, Costa Rica
}

\section{Identification of four species of Clupeiformes (Actinopterygii) through analysis of mitochondrial DNA sequences in fishing exploitation areas of the Gulf of Nicoya, Costa Rica}

\author{
Carolina Sancho-Blanco ${ }^{1}$, Luis Hernández-Noguera ${ }^{2}$, Luis Vega-Alpízar ${ }^{2}$, Rosa Soto-Rojas ${ }^{2}$ y \\ Rodolfo Umaña-Castro ${ }^{1 *}$
}

\begin{abstract}
RESUMEN
Las sardinas junto con las anchovetas constituyen unos de los grupos de mayor importancia en la pesquería del golfo de Nicoya. Son alimento para peces de mayor tamaño y aves marinas, por tanto, no solo sufren explotación pesquera, sino también presentan altas tasas de mortalidad natural. Debido a la importancia comercial y ecológica que representan estas especies, es necesario contar con una correcta identificación taxonómica, principalmente para la elaboración de estudios pesqueros. Antes, la determinación de especies de peces se fundamentaba únicamente en características morfológicas externas, sin embargo, no siempre es posible, debido a su similitud morfológica, por lo que el uso combinado de datos morfológicos junto con los genéticos basados en genes mitocondriales puede contribuir en su reconocimiento. Históricamente, estudios taxonómicos han agrupado a las especies $O$. libertate, $O$. medirastre y $O$. bulleri como integrantes del complejo Opisthonema spp., dada la gran similitud de forma. Mientras, para la familia Engraulidae, las indagaciones taxonómicas son escasas. En el presente trabajo, se identificaron molecularmente especies del complejo Opisthonema spp. y una especie del género Cetengraulis sp., colectadas entre junio y diciembre del 2017, mediante secuenciación de genes mitocondriales: COI, Ctyb y $16 \mathrm{~S}$ ARNr. Los hallazgos confirman, con datos moleculares, la identidad y presencia de tres especies del complejo Opisthonema spp. y una especie de anchoveta,
\end{abstract}

1 Universidad Nacional. Escuela de Ciencias Biológicas, Laboratorio de Análisis Genómico (LAGEN).

carolina.sancho.blanco@una.ac.cr, ORCID: https://orcid.org/0000-0002-0378-001X

rodolfo.umana.castro@una.ac.cr*, ORCID: https://orcid.org/0000-0003-0041-2788

2 Universidad Nacional. Estación de Biología Marina Juan Bertoglia Richards, Escuela de Ciencias Biológicas,

Laboratorio de Análisis Biológicos-Pesqueros.

luis.hernandez.noguera@una.ac.cr, ORCID: http://orcid.org/0000-0003-3698-7161

luigivega3@gmail.com, ORCID: https://orcid.org/0000-0002-1993-0705

rosa.soto.rojas@una.ac.cr, ORCID: http://orcid.org/0000-0002-1928-2023 
localizadas en zonas de explotación pesquera en el golfo de Nicoya, Costa Rica, información que facilita el uso y manejo de estos recursos pesqueros en un área marina de importancia comercial en el país.

Palabras clave: Opisthonema spp., Cetengraulis sp., ADNmt, ADNr, posicionamiento taxonómico

\begin{abstract}
Sardines, together with anchovies, constitute one of the most important groups in the fishery of the Gulf of Nicoya. They are consumed by large fish and seabirds; therefore, they not only suffer from fishing exploitation but also have high rates of natural mortality. Due to their commercial and ecological importance, it is necessary to have a reliable taxonomic identification of these species, mainly for the preparation of fisheries studies. Previously, the identification of fish species was based only on external morphological characteristics; however, this is not always possible due to their morphological similarity, but the combined use of morphological and genetic data based on mitochondrial genes can contribute to their accurate recognition. Historically, taxonomic studies have grouped the species $O$. libertate, O. medirastre and O. bulleri as members of the Opisthonema spp. complex due to their great morphological similarity, while, there are few taxonomic studies of the family Engraulidae. In the present work, species of the Opisthonema spp. complex and a species of the genus Cetengraulis sp., collected between June-December 2017, were molecularly identified by sequencing mitochondrial genes: COI, Ctyb, and 16S rRNA. The findings of the molecular data analysis confirm the identity and presence of three species of the Opisthonema spp. complex and a species of anchovy, in areas of fishing exploitation in the gulf of Nicoya, Costa Rica, information that facilitates the use and management of these fishing resources in a marine area of commercial importance for the country.
\end{abstract}

Keywords: Opisthonema spp., Cetengraulis $s p .$, mtDNA, rDNA, taxonomic placement

\section{INTRODUCCIÓN}

El orden Clupeiformes incluye especies conocidas como arenques, sardinas y anchovetas, todas de gran importancia comercial para las pesquerías globales. Asimismo, abarca familias como Chirocentridae, Clupeidae, Denticipitidae, Dussumieriidae, Engraulidae Pristigasteridae y Sundasalangidae; sin embargo, la taxonomía de especies de las familias Clupeidae y Engraulidae aún sigue siendo controversial en términos morfológicos y genéticos ( $\mathrm{Li}$ \& Ortí, 2007; Bloom \& Lovejoy, 2014; Vicente et al. 2020).

Tanto los Clupeidos como los Engraulidos son individuos de tallas pequeñas generalmente, se agrupan en grandes cardúmenes, son filtradores y conforman la base de la cadena alimenticia de los ecosistemas (Fischer et al. 1995); por tanto, no solo sufren de la explotación pesquera, sino que también presentan altas tasas de mortalidad natural, al ser el alimento para 
otros organismos como peces de mayor tamaño y aves marinas (Bussing \& López, 1994).

En las costas del Pacífico, el género Opisthonema Gill, 1861 está representado por cuatro especies: Opisthonema libertate Günther, 1867, Opisthonema medirastre Berry \& Barrett, 1963, Opisthonema bulleri Regan, 1904 y Opisthonema berlangai Berry \& Barrett, 1963 (Froese \& Pauly, 2019). En Costa Rica, debido a su difícil diferenciación morfológica, históricamente las especies $O$. libertate, medirastre y bulleri se han agrupado como integrantes del complejo Opisthonema spp. y han sido utilizadas para la industria de enlatados con un mismo valor comercial (Soto \& Rodríguez, 1999; Vega-Corrales, 2010). Por su parte, la familia Engraulidae se encuentra representada, principalmente, por Cetengraulis mysticetus, el cual es un recurso pesquero de importancia, al emplearse como carnada viva por la flota pesquera artesanal en la zona interna del golfo de Nicoya, Costa Rica (Rodríguez \& Gómez, 1998; Soto \& Rodríguez, 1999; Murase et al. 2014).

Estudios enfocados en la identificación y taxonomía del género Opisthonema señalan si las diferencias encontradas en el nivel morfológico son el resultado de variaciones intraespecíficas o de características diagnósticas (Pérez-Quiñónez et al. 2017). Por otro lado, para la familia Engraulidae, los hallazgos en cuanto a taxonomía molecular son escasos, a pesar de su importancia pesquera y del impacto ecológico potencial que las pesquerías pueden tener en el ecosistema pelágico (Wang et al. 2018). A partir del desarrollo de técnicas moleculares, se inició la incorporación de datos genéticos, basados en el ADN mitocondrial y nuclear, que permitieran esclarecer interrogantes taxonómicas no resueltas mediante características morfológicas y merísticas (Ward et al. 2005; Teletchea, 2009; Ardura et al. 2010; Carvalho et al. 2011; Pereira et al. 2013; Pérez-Quiñónez, 2014; Durand et al. 2017; Pérez-Quiñónez et al. 2017; Goodbody-Gringley et al. 2019; Pérez-Quiñónez et al. 2019).

En peces, los marcadores genéticos más utilizados para la identificación de especies se basan en regiones del ADN mitocondrial (Jahan et al. 2017), los cuales, al mostrar alta tasa de mutación y gran variabilidad interespecífica, hacen posible una adecuada distinción entre especies (Rocha et al. 2005; Paine et al. 2007). Entre dichos marcadores mitocondriales, se encuentran el gen del 16S del ARN ribosómico (16S ARNr), el gen del citocromo oxidasa I (COI), el gen citocromo oxidasa $b(\mathrm{Cytb})$, entre otros. El 16S ARNr es altamente conservado; variaciones de unos pocos nucleótidos en su estructura permiten inferir relaciones taxonómicas en diferentes niveles, inclusive entre taxa estrechamente relacionados (Chakraborty \& 
Iwatsuki, 2006). Por su parte, el gen COI, a pesar de ser muy conservado, posee regiones polimórficas que facilitan la identificación de especies (Hebert et al. 2003). Mientras, la secuencia de nucleótidos del gen Cytb contiene información específica de la especie; posibilita reconstruir tanto relaciones inter- o intraespecíficas como de nivel superior, por lo cual se considera un gen con alto rendimiento en filogenia molecular (Peng et al. 2004).

La identificación taxonómica de estas especies en las capturas, a través de los diferentes artes de pesca en el golfo de Nicoya, permite evaluar el estado actual de sus poblaciones; fijar la cuota de pesca, el establecimiento correcto de las vedas; recomendar medidas de ordenamiento y pautas de captura, así como para garantizar los niveles de biomasa del stock desovante (Kochzius et al. 2010; Pérez-Quiñónez et al. 2018). De esta forma, se mantiene, además, la sostenibilidad de otras especies de interés pesquero -corvinas, pargos, jureles- y ecológico -las aves- (FAO, 2018).

En el presente estudio, se identificó, molecularmente, a partir de secuencias del ADN mitocondrial (ADNmt) junto con caracteres morfológicos, algunas especies que integran las familias Engraulidae y Clupeidae. Con ello, se proporcionan elementos suficientes que instan a diferenciar entidades taxonómicas en una zona de explotación pesquera en el golfo de Nicoya, Costa Rica. La información obtenida facilitará el uso y manejo de estos recursos pesqueros en una zona marina de importancia comercial en el país, al detectar posibles sesgos en la identificación tradicional basada en caracteres morfológicos diagnósticos.

\section{MATERIALES Y MÉTODOS}

\section{Obtención de las muestras}

Se recolectaron especímenes del complejo Opisthonema spp. y $\mathrm{Ce}$ tengraulis $s p$. distribuidos específicamente en el golfo de Nicoya, Pacífico Central, Costa Rica, de junio a setiembre de 2017. Se identificaron, en la zona externa del golfo, individuos de Opisthonema bulleri (sardina azul), $O$. medirastre y $O$. libertate (sardina gallera), en un rango de longitudes estándar de $137 \mathrm{~mm}$ a $184 \mathrm{~mm}$. Por otra parte, en la zona interna del golfo, se detectaron individuos de C. mysticetus (anchoveta) (Fig. 1). La identificación preliminar se realizó a partir de caracteres morfológicos diagnósticos, utilizando la metodología previamente descrita por Berry \& Barret, 1963; Bussing \& López, 1994, Robertson \& Allen, 2002; Pérez Quiñones, 2014. (Cuadro 1). 


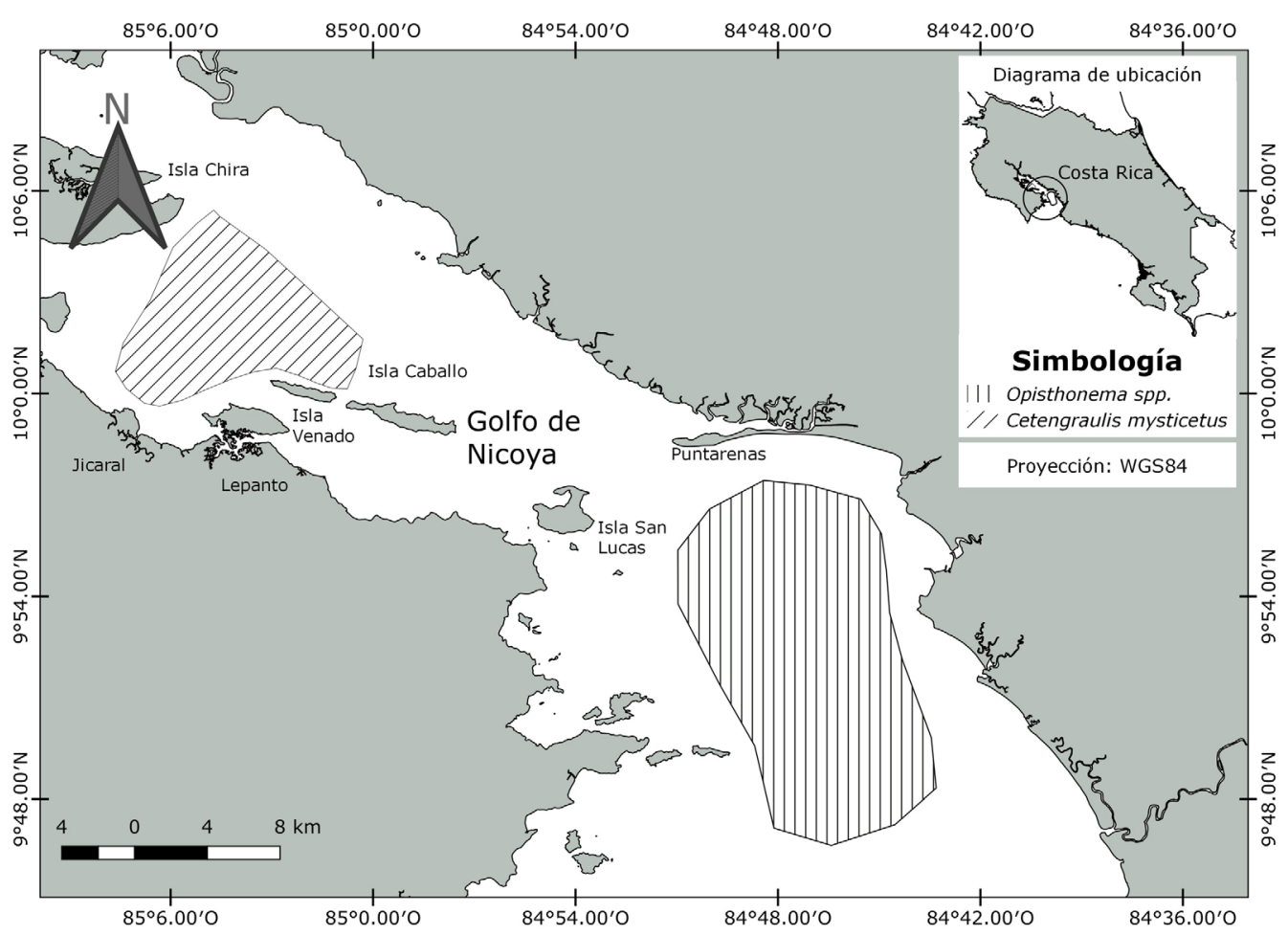

Fig. 1. Áreas de captura de las especies analizadas. Fuente de datos de figura: Atlas 2014 (TEC) y Laboratorio de Análisis Biológicos Pesqueros (UNA) (QGIS, 2018)

Fig. 1. Areas in which the species analyzed were captured. Data source: Atlas 2014 (TEC) and Fisheries Biological Analysis Laboratory (UNA) (QGIS, 2018)

Extracción de ADN, amplifi- Kit (Thermo Fisher Scientific), bajo caciones y secuenciación de genes mitocondriales

Se realizó una extracción de ADN total de especies del complejo Opisthonema spp., O. medirastre $(\mathrm{n}=11), O$. libertate $(\mathrm{n}=4)$, O. bulleri $(\mathrm{n}=7)$ y de la familia Engraulidae C. mysticetus $(\mathrm{n}=5)$, a partir de $30 \mathrm{mg}$ de tejido, aproximadamente, mediante el kit comercial PureLink Genomic DNA Mini las recomendaciones del fabricante. La cantidad de muestras seleccionadas por especie, en este estudio, se respalda en un trabajo previamente realizado por Bingpeng et al. 2018. El ADN obtenido fue amplificado mediante una PCR punto final (volumen final: 25 $\mu \mathrm{L}$ ) compuesta por $1 \mathrm{X}$ de PCR Máster Mix, $0.9 \mu \mathrm{M}$ de cada cebador y alrededor de $100 \mathrm{ng}$ de ADN. Se analizaron 
Cuadro 1. Características morfológicas diagnósticas evaluadas para las especies de Opisthonema spp. analizadas en este estudio para la localidad del Golfo de Nicoya, Costa Rica

Table 1. Diagnostic morphological characteristics evaluated for Opisthonema spp., analyzed in this study for the location of the Gulf of Nicoya, Costa Rica

\begin{tabular}{|c|c|c|c|c|c|c|}
\hline Especie & Código & $\begin{array}{c}\text { bc } \\
\text { (unidades) }\end{array}$ & Media bc & std (mm) & Media std & ph \\
\hline \multirow{9}{*}{ O. medirastre } & GN04 & 70 & \multirow{9}{*}{68.0} & 168 & \multirow{9}{*}{163.6} & 1 \\
\hline & GN10 & 63 & & 164 & & / \\
\hline & GN11 & 78 & & 184 & & 1 \\
\hline & GN20 & 71 & & 172 & & / \\
\hline & GN24 & 62 & & 169 & & 1 \\
\hline & GN28 & 75 & & 179 & & / \\
\hline & PS19 & 62 & & 140 & & / \\
\hline & $\mathrm{PS} 23$ & 69 & & 166 & & / \\
\hline & PS29 & 64 & & 137 & & / \\
\hline \multirow{3}{*}{ O. libertate } & PS04 & 88 & \multirow{3}{*}{86.3} & 151 & \multirow{3}{*}{144.9} & + \\
\hline & PS11 & 85 & & 142 & & + \\
\hline & $\mathrm{PS} 28$ & 86 & & 142 & & + \\
\hline \multirow{6}{*}{ O. bulleri } & GN01 & 41 & \multirow{6}{*}{36.6} & 159 & \multirow{6}{*}{157.9} & / \\
\hline & GN02 & 37 & & 158 & & / \\
\hline & GN03 & 34 & & 157 & & / \\
\hline & GN04 & 35 & & 163 & & / \\
\hline & GN06 & 37 & & 164 & & / \\
\hline & GN12 & 36 & & 147 & & 1 \\
\hline
\end{tabular}

bc: branquiespinas ceratobranquiales, std: longitud estándar, ph: pecas humerales (ausentes $=/$, presentes $=+$ )

los genes mitocondriales citocromo oxidasa subunidad I (COI) y citocromo b (Cytb), así como el gen del ARN ribosomal 16S (ARNr 16S) (Cuadro 2). La PCR fue llevada a cabo en las siguientes condiciones: $94^{\circ} \mathrm{C}$ por $3 \mathrm{~min}$ (desnaturalización inicial); 40 ciclos de $94^{\circ} \mathrm{C}$ por $30 \mathrm{~s}$ (desnaturalización), $48 / 55^{\circ} \mathrm{C}$ por $30 \mathrm{~s}$ (alineamiento), $72^{\circ} \mathrm{C}$ por $45 \mathrm{~s}$ (extensión) $\mathrm{y}$, finalmente, $72^{\circ} \mathrm{C}$ por 10 min como extensión final. Los productos de PCR se resolvieron por electroforesis en gel de agarosa al $1.5 \%$. La corrida se ejecutó a $80 \mathrm{~V}$ por 
60 min en una solución amortiguadora TBE $1 \mathrm{X}(\mathrm{pH} 8.0)$. Los amplicones obtenidos de los tres genes fueron secuenciados en ambas direcciones, utilizando la secuenciación de Sanger en un analizador genético ABI 3130, bajo una química de secuenciación BigDye Terminator v3.1.

Cuadro 2. Cebadores utilizados en este estudio y sus respectivas secuencias de ADN. Se indica la región mitocondrial (mt) objetivo, las secuencias del cebador en dirección 5'-3', la referencia (Ref.) y el tamaño esperado del amplicón (Te) en pares de bases (pb) Table 2. Primers used in this study and their respective DNA sequences. Target mitochondrial region (mt), 5'-3' primer sequences direction, reference (Ref.), and expected amplicon size (Te) in base pairs (bp) are indicated

\begin{tabular}{|c|c|c|c|c|}
\hline Organismo & $\begin{array}{c}\text { Región génica } \\
\text { (mt) }\end{array}$ & Secuencia $\left(5^{\prime}-3^{\prime}\right)$ & Ref. & Te (pb) \\
\hline \multirow{3}{*}{ Opisthonema } & $16 \mathrm{~S}$ & $\begin{array}{l}\text { 5'CGCCTGTTTAACAAAAA- } \text { CAT3' } \\
\text { 5'CCGGTTTGAACTCAGAT- }^{\prime} \\
\text { CACGT3' }\end{array}$ & $\begin{array}{l}\text { Palumbi, } \\
1996 .\end{array}$ & $\sim 520$ \\
\hline & $\mathrm{COI}$ & $\begin{array}{l}\text { 5'TCAACYAATCAYAAAGA- } \\
\text { TATYGGCAC3' } \\
\text { 5'ACTTCYGGGTGRCCRAA- }^{\prime} \text { 'RAATCA3' }\end{array}$ & $\begin{array}{l}\text { Baldwin et } \\
\text { al. } 2009 .\end{array}$ & $\sim 651$ \\
\hline & Cytb & $\begin{array}{l}\mathbf{5}^{\prime} \text { AAACTGCAGCCCCTCA- } \\
\text { GAATGATATTTGTCCTCA3' } \\
\text { 5'AAAAAGCTTCCATCCAA- }^{\prime} \text { ' } \\
\text { CATCTCAGCATGATGAAA3' } \\
\text { 5'CGAAGCTTGATAT- }^{\prime} \\
\text { GAAAAACCATCGTTG3' }\end{array}$ & $\begin{array}{l}\text { Kocher et } \\
\text { al. } 1989 \text {, } \\
\text { Meyer et al. } \\
1990 .\end{array}$ & $\sim 370$ \\
\hline \multirow{3}{*}{ Engraulidae } & $16 \mathrm{~S}$ & $\begin{array}{l}\text { 5'CGCCTGTTTAACAAAAA- }^{\prime} \\
\text { CAT3' } \\
\text { 5'CCGGTTTGAACTCAGAT- }^{\prime} \text { 'CACGT3' }\end{array}$ & $\begin{array}{l}\text { Palumbi, } \\
1996 .\end{array}$ & $\sim 520$ \\
\hline & Cytb & $\begin{array}{l}\text { 5'TGACTTGAAAAACCACC- } \\
\text { GTTGTTATTCAAC3' } \\
\text { 5'CTAGCTTTGGGAGYTAGD- }^{\prime} \text { 'GGTGGRAGTT3' }\end{array}$ & $\begin{array}{l}\text { Bloom \& } \\
\text { Lovejoy } \\
2012 .\end{array}$ & $\sim 1100$ \\
\hline & $\mathrm{COI}$ & $\begin{array}{l}\text { 5'TTCTCAACCAACCACAAA- } \\
\text { GACATTGG3' } \\
\text { 5'TAGACTTCTGGGTGGC- }^{\prime} \\
\text { CAAAGAATCA3' }\end{array}$ & $\begin{array}{l}\text { Ivanova et } \\
\text { al. } 2006 \\
\text { Ward et al. } \\
2005 \text {. }\end{array}$ & $\sim 620$ \\
\hline
\end{tabular}


Análisis molecular y de posicionamiento taxonómico

Utilizando el programa Geneious versión R9 (Biomatters), se editaron las secuencias nucleotídicas obtenidas, mediante una edición manual de los bordes no alineados. Luego, para corroborar, preliminarmente, su identidad taxonómica en el nivel de género, esas secuencias fueron evaluadas en el programa en línea BLASTn (Altschul et al. 1990), disponible en el National Center for Biotechnology Information (NCBI) (Benson et al. 2012); al final, fueron depositadas en el GenBank (Cuadro 3 ). Por otra parte, se realizó un alineamiento múltiple de secuencias, usando el programa MAFFT 7.0 (Katoh et al. 2009) y aplicando el método iterativo de refinamiento G-INS-i (1PAM/k = 2). Para determinar el mejor modelo de sustitución nucleotídica, se analizó el alineamiento múltiple mediante el JModelTest v2.1.10 (Darriba et al. 2012), empleando parámetros por defecto, bajo el criterio de información de Akaike con $95 \%$ de intervalo de confianza y tomando en cuenta la corrección AICc. El programa arrojó los seis mejores modelos para las matrices correspondientes a los genes $16 \mathrm{~S}$ ARN ribosomal [GTR + G, GTR + I, GTR + $\mathrm{I}+\mathrm{G}, \mathrm{TVM}+\mathrm{I}, \mathrm{TVM}+\mathrm{I}+\mathrm{G}$, TIM3 $+\mathrm{G}]$, citocromo oxidasa subunidad 1 [TVM + G, TPM2uf + G, TVM + I + G, TPM2uf + I + G, GTR + G, TIM2 + $\mathrm{G}]$, para el cual TVM $+\mathrm{G}=\mathrm{GTR}+\mathrm{G}$, y citocromo oxidasa $b$ [TrN + G, TIM1 $+\mathrm{G}, \mathrm{TrN}+\mathrm{I}+\mathrm{G}, \mathrm{TIM} 3+\mathrm{I}+\mathrm{G}, \mathrm{TIM} 2$ $+\mathrm{I}+\mathrm{G}, \mathrm{GTR}+\mathrm{G}]$, para el cual $\mathrm{TrN}+$ $\mathrm{G}=\mathrm{GTR}+\mathrm{G}$. La elaboración de las topologías multigénicas de posicionamiento taxonómico se realizó mediante dos métodos: el primero, utilizando el programa raxmlGUI v.7.4.2 (Stamatakis et al. 2005), bajo un algoritmo de máxima verosimilitud (ML), con un modelo reversible en tiempo general (GTR-GAMMA) y aplicando 5000 permutaciones como valor bootstrap. El segundo método se efectuó a través de inferencia bayesiana (BI), con el programa MrBayes v.3.2.6 (Huelsenbeck \& Ronquist, 2001), un modelo de sustitución GTR-GAMMA y los siguientes parámetros: $\mathrm{nst}=6$, rates $=$ gamma, cuatro cadenas simultáneas de Markov Monte Carlo $(\mathrm{mcmc})$ y ngen $=$ 1000000. La visualización y edición de los árboles obtenidos se confeccionó con el programa FigTree v1.4 (Rambaut, 2009); después, se concatenaron manualmente, según la similitud de las topologías y utilizando secuencias nucleotídicas ya reportadas en la base de datos del NCBI (Benson et al. 2012).

\section{RESULTADOS}

Las características morfológicas diagnósticas evaluadas para las tres especies del género Opisthonema spp. muestran que su identificación, tras observar las pecas humerales, permite 
distinguir $O$. libertate (presencia de pecas humerales, ph) de O. medirastre y $O$. bulleri (ausencia de ph); sin embargo, este análisis se debe realizar con el espécimen fresco o recién capturado, de lo contrario, se dificulta su diferenciación. Por otro lado, medir la longitud estándar (std) y el conteo de las unidades de branquiespinas ceratobranquiales (bc) muestra una media de 86.3 para $O$. libertate $(\mathrm{n}=3) ; 68.0, O$. medirastre $(\mathrm{n}=9)$, y 36.6, O. bulleri $(n=6)$. Estos resultados se encuentran entre los intervalos de unidades bc y longitud estándar señalados en la literatura (Cuadro 1).

A partir de las reacciones de PCR, fue posible obtener amplificaciones positivas (tamaño esperado del amplicón en pares de bases), para los tres cebadores utilizados (16S, COI y Cytb), en las especies $O$. medirastre, $O$. libertate y $O$. bulleri del complejo Opisthonema spp., así como para una especie de la familia Engraulidae. Los amplicones recuperados se purificaron y las secuencias parciales conseguidas se depositaron en el Genbank (Cuadro 3).

Cuadro 3. Secuencias depositadas en el Genbank (https://www.ncbi.nlm.nih.gov/ genbank) de las tres especies del complejo Opisthonema spp., derivadas de tres marcadores moleculares y una especie del género Cetengraulis $s p$. obtenidas de este estudio

Table 3. Sequences deposited on Genbank (https://www.ncbi.nlm.nih.gov/genbank) of three species of Opisthonema spp. complex, derived from three molecular markers and one species from the genus Cetengraulis $s p$. obtained from this study

\begin{tabular}{|c|c|c|c|c|c|}
\hline \multirow{2}{*}{ Especie } & \multirow{2}{*}{ Código } & \multirow{2}{*}{$\begin{array}{l}\text { Localización } \\
\text { en Golfo de } \\
\text { Nicoya }\end{array}$} & \multicolumn{3}{|c|}{ Números de accesión GenBank } \\
\hline & & & $16 S$ & COI & Cytb \\
\hline \multirow{12}{*}{ O. medirastre } & Om_GN04 & \multirow{12}{*}{ Zona externa } & MG889827 & MN329855 & MK499391 \\
\hline & Om_GN10 & & MG889828 & MN329857 & MK499395 \\
\hline & Om_GN11.1 & & MG889829 & MN329858 & MK499390 \\
\hline & Om_GN11.2 & & / & MN329865 & / \\
\hline & Om_GN20 & & / & MN329853 & MK499388 \\
\hline & Om_GN24 & & MG889830 & MN329863 & MK499396 \\
\hline & Om_GN28 & & / & MN329850 & MK499389 \\
\hline & Om_PS19 & & MG889831 & MN329854 & MK499393 \\
\hline & Om_PS23 & & MG889832 & MN329860 & MK499394 \\
\hline & Om_PS29.1 & & MG889833 & MN329859 & / \\
\hline & Om_PS29.2 & & / & MN329861 & / \\
\hline & $\begin{array}{l}\text { Om_voucher02 } \\
\text { (UCR-3194.002) }\end{array}$ & & / & l & MK499392 \\
\hline
\end{tabular}




\begin{tabular}{|c|c|c|c|c|c|}
\hline \multirow{2}{*}{ Especie } & \multirow{2}{*}{ Código } & \multirow{2}{*}{$\begin{array}{l}\text { Localización } \\
\text { en Golfo de } \\
\text { Nicoya }\end{array}$} & \multicolumn{3}{|c|}{ Números de accesión GenBank } \\
\hline & & & $16 S$ & COI & Cytb \\
\hline \multirow{4}{*}{ O. libertate } & Ol_PS04 & \multirow{4}{*}{ Zona externa } & MG889834 & MN329856 & MK499398 \\
\hline & Ol_PS11 & & MG889835 & MN329864 & MK499397 \\
\hline & Ol_PS28 & & l & MN329851 & l \\
\hline & $\begin{array}{l}\text { Ol_voucher01 } \\
\text { (UCR-3194.001) }\end{array}$ & & I & MN329862 & l \\
\hline \multirow{7}{*}{ O. bulleri } & Ob_GN01 & \multirow{7}{*}{ Zona externa } & MG889836 & MN329866 & MK499400 \\
\hline & Ob_GN02 & & MG889837 & MN329847 & l \\
\hline & Ob_GN03 & & MG889838 & MN329848 & l \\
\hline & Ob_GN04 & & MG889839 & MN329867 & MK499401 \\
\hline & Ob_GN06 & & l & MN329849 & l \\
\hline & Ob_GN12 & & l & MN329852 & MK499399 \\
\hline & $\begin{array}{l}\text { Ob_voucher03 } \\
\text { (UCR-3194.003) }\end{array}$ & & I & l & MK499402 \\
\hline \multirow{5}{*}{ C. mysticetus } & Cm_GNzi 02 & \multirow{5}{*}{ Zona interna } & l & MN329846 & MK499404 \\
\hline & Cm_GNzi 02.1 & & l & 1 & MK499407 \\
\hline & Cm_GNzi_04 & & l & MN329845 & MK499403 \\
\hline & Cm_GNzi 06 & & l & MN329844 & MK499405 \\
\hline & Cm_GNzi 06.1 & & l & I & MK499406 \\
\hline
\end{tabular}

Las topologías de los árboles filogenéticos construidos con base en regiones parciales de los genes Cytb, COI y $16 \mathrm{~S}$ (Figuras 2A, 2B, 2C) confirman la existencia, en el golfo de Nicoya, de un grupo monofilético conformado por tres clados genéticamente diferentes, según la alta divergencia de las agrupaciones formadas. Tales clados se definen como $O$. medirastre, $O$. libertate y $O$. bulleri; así, se demuestra la presencia de las tres especies que componen el complejo complejo Opisthonema spp. en el golfo de Nicoya, Puntarenas. Además, se observa que para los genes Cytb y $16 \mathrm{~S}$ las especies $O$. libertate y $O$. medirastre son similares entre sí a nivel genético. Estos resultados moleculares son congruentes con los datos morfométricos y merísticos obtenidos, acorde con las características diagnósticas (pecas humerales y branquiespinas ceratobranquiales) indicadas por Bussing y Lopez (1994). Por otro lado, los individuos de $C$. mysticetus, analizados mediante marcadores mitocondriales, se posicionan 
Identificación de cuatro especies de Clupeiformes (Actinopterygii) mediante análisis de secuencias de $\mathrm{ADN}$ mitocondrial en zonas de explotación pesquera del Golfo de Nicoya, Costa Rica

taxonómicamente en el clado marino demostrando la especificidad en el Cetengraulis: Cetegraulis edentulus método de identificación taxonómica y $C$. mysticetus, separando en ramas con herramientas moleculares. bien soportadas a los especímenes originarios del Pacífico y del Atlántico,
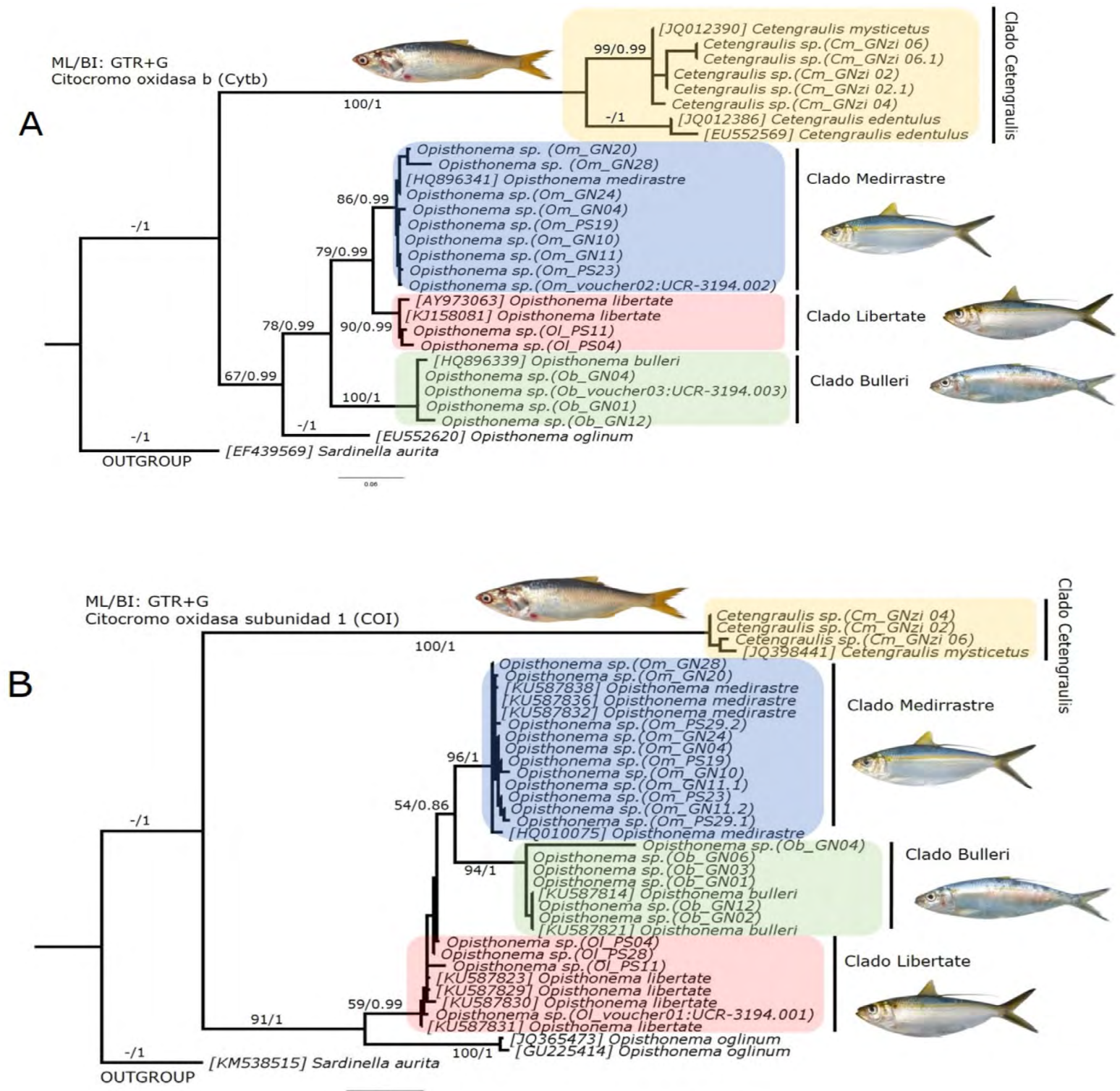


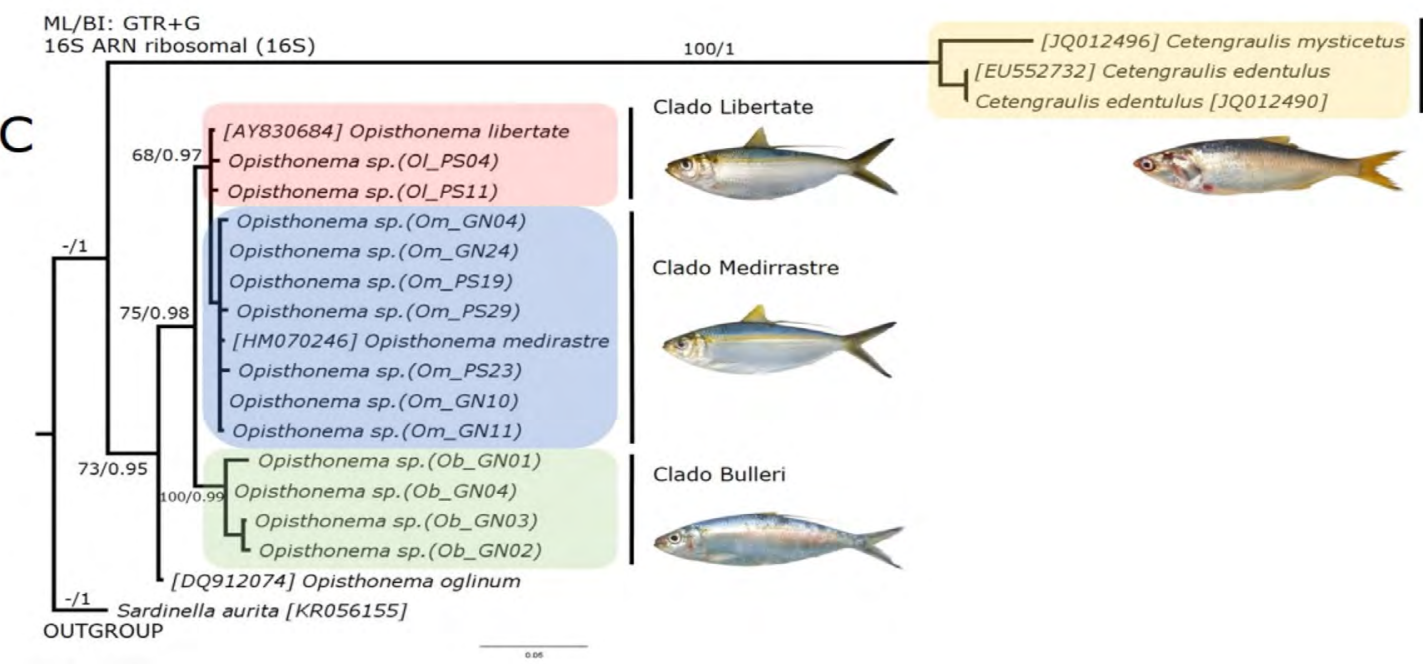

Fig. 2. Topologías de posicionamiento taxonómico mediante Máxima Verosimilitud/ Inferencia Bayesiana (ML/BI), entre secuencias genéticas de Opisthonema spp., Cetengraulis sp. del Pacífico costarricense y secuencias parciales de los genes A) citocromo b (Cytb), B) citocromo oxidasa subunidad 1 (COI) y C) 16S ARN ribosomal $(16 \mathrm{~S})$, obtenidas de la base de datos Genbank. El número en las ramas indica el valor de soporte boostrap (porcentaje derivado de 5000 permutaciones para ML y 1000 000 generaciones MCMC para BI). Sardinella aurita es definido como grupo externo. La región y el código de colecta de las muestras de este estudio se presentan entre paréntesis. Para las secuencias obtenidas del Genbank, los números de accesión se muestran entre corchetes. Valores de bootstrap o de probabilidad posterior menor a $50 \%$ o no obtenidos no se muestran en la topología o se indican con -

Fig. 2. Taxonomic placement topologies using Maximum Likelihood / Bayesian Inference (ML / BI), between genetic sequences of Opisthonema spp., Cetengraulis $s p$. from the Costa Rican Pacific and partial sequences of the genes A) cytochrome b (Cytb), B) cytochrome oxidase subunit 1 (COI) and C) 16S ribosomal RNA (16S), obtained from the Genbank database. The number in the branches indicates the bootstrap support value (percentage derived from 5000 permutations for ML and 1 000000 MCMC generations for BI). Sardinella aurita is defined as an external group. The region and collection code of the samples in this study are shown in parentheses. For sequences obtained from the Genbank, the accession numbers are shown in square brackets. Boostrap or posterior probability values less than $50 \%$ or not obtained are not shown in the topology or indicated with a - 


\section{DISCUSIÓN}

Según Berry y Barret (1963), las diferenciaciones taxonómicas de estas especies se basan, principalmente, en dos aspectos: distribución geográfica y número o cantidad de branquiespinas de la región ceratobranquial (bc), asociadas a la longitud del individuo. Se reporta que $O$. libertate presenta 71-107 bc; O. medirastre, 50-68 bc, y $O$. bulleri, $28-35$ bc. Posteriormente, en un estudio realizado por Rodríguez-Sánchez (1985), se considera que la densidad de branquiespinas disminuye en relación con el tamaño del espacio interbranquial, hecho que discrepa de la veracidad de los caracteres merísticos descritos por Berry y Barret (1963). Fue, entonces, menor la cantidad de branquinespineas en $O$. bulleri, seguido de O.medirastre, y mayor en O.libertate, por lo que se concluye que el conteo de las branquiespinas permite identificar únicamente $O$. libertate y O. bulleri.

En este estudio, el conteo de las branquiespinas de la región ceratobranquial concuerda con los rangos de las unidades bc para cada especie del complejo Opisthonema spp., señaladas en la literatura. De la misma forma, los valores medidos de la longitud estándar se encuentran entre los límites descritos para las especies en estudio, lo que facilita la identificación en especímenes recién capturados (Cuadro 1).
Por otro lado, la presencia de las pecas humerales permitió distinguir, con mayor facilidad, entre los especímenes de $O$. libertate y los de $O$. medirastre y O. bulleri.

Una investigación realizada por Lagúnez y Rodríguez (1992), en poblaciones de estas mismas especies en el pacífico mexicano, a partir de análisis de proteinogramas, arrojó la existencia de seis patrones en $O$. libertate muy similares a $O$. medirastre; mientras, estos difieren de los de O. bulleri, por lo tanto, los autores consideran que las dos primeras especies son entidades taxonómicamente muy cercanas y que más que especies distintas podrían ser subpoblaciones de una misma especie. Por su parte, Pérez-Quiñónez (2014), a través de patrones morfométricos de las tres especies y un análisis de componentes principales mediante variables canónicas, obtuvo una mayor similitud entre las formas de $O$. medirastre y $O$. libertate, mientras que $O$. bulleri presentaba más diferenciación con respecto a la forma corporal. Nuestros hallazgos muestran una mayor cercanía filogenética entre $O$. libertate y $O$. medirastre, de acuerdo con las topologías taxonómicas obtenidas para los genes 16S y Cytb, lo cual concuerda con lo reportado por los autores citados anteriormente.

La adición de datos moleculares en estudios morfológicos mejora significativamente el soporte y la 
resolución de los análisis filogenéticos (Wortley \& Scotland, 2006); con ello, proporciona datos relevantes cuando se evalúan especies de interés comercial. En estas últimas, no es posible (o resulta complejo) determinar las capturas por especie mediante características morfológicas (PérezEnríquez et al. 2016), como es el caso de las sardinas de la familia Clupeidae, razón por la cual dichas capturas son registradas, generalmente, como sardina crinuda (Opisthonema spp.) tanto en Costa Rica como en otros países de la región -México entre ellos- (Pérez-Quiñonez et al. 2020). Lo anterior conduce a una posible subestimación o sobreestimación de la riqueza de especies e incluso a la sobreexplotación de las poblaciones de peces.

Li y Ortí (2007), a partir de análisis de máxima parsimonia, máxima verosimilitud y análisis bayesianos de datos con ADN mitocondrial (12S y $16 \mathrm{~S}$ ) y ADN nuclear (genes activadores de recombinación, RAG 1 y RAG 2), encontraron monofilia en subfamilias Engraulidae (Engraulis y Anchoa), pero no en la familia Clupeidae. Pérez-Quiñónez et al. (2017), completaron análisis moleculares del gen citocromo oxidasa I (COI) del ADNmt para especies reconocidas del complejo Opisthonema spp., en la región sur del golfo de California, México, y con fundamento en sus resultados morfológicos y genéticos se soporta la existencia de tres entidades evolutivas para la región: $O$. bulleri, $O$. medirastre y $O$. libertate. Estos datos coinciden con nuestros hallazgos, de acuerdo con la topología observada en el árbol de posicionamiento taxonómico para los tres genes (Cytb, COI y 16S ARNr), lo que apoya la identidad y existencia de las tres especies para la región y, específicamente, para el golfo de Nicoya, Costa Rica.

La correcta identificación de estas especies de interés pesquero, mediante características morfológicas y genéticas, facilita el uso y manejo de estos recursos marinos dentro de una zona de relevancia comercial en el país como lo es el golfo de Nicoya. Ello con el objetivo de minimizar la alteración del porcentaje de la biomasa virginal que debe contener el ecosistema y así mantener el resto de los eslabones de la cadena alimentaria.

\section{CONCLUSIÓN}

Con base en los resultados obtenidos en este estudio sobre los caracteres morfométricos y merísticos diagnósticos, así como en herramientas moleculares, se confirma la identificación de tres entidades taxonómicas diferentes en el complejo Opisthonema spp. descrito en el golfo de Nicoya: $O$. libertate, O. medirastre y $O$. bulleri, confirmando su identidad y presencia en sitios de pesca en el exterior de dicho golfo. Por otro lado, se corrobora 
el posicionamiento taxónomico de los individuos de C. mysticetus en el clado marino Cetengraulis sp., C. edentulus y C. mysticetus, lo cual comprueba su identidad y presencia en los sitios de pesca dentro del golfo de Nicoya. Con la información obtenida en este trabajo y la disponible en la base mundial de datos genéticos, es posible realizar, a futuro, investigaciones comparativas de diversidad y estructura genética (por ejemplo, redes de haplotipos y RAD-seq), en el golfo de Nicoya y otras zonas de captura de Clupeidos de importancia comercial.

\section{AGRADECIMIENTOS}

Al proyecto 0079-14: Aportes a la gestión pesquera artesanal y semi-industrial del golfo de Nicoya, Costa Rica, financiado por la Ley de Pesca, Costa Rica. También, a Karolina Ramos Jiménez y Karen Oviedo Bolaños, por el soporte técnico en los ensayos moleculares. Por último, a los evaluadores, por sus recomendaciones y aportes para la publicación de este artículo.

\section{REFERENCIAS}

Altschul, S. F., Gish, W., Miller, W., Myers, E. W. \& Lipman, D. J. (1990). Basic local alignment search tool. J. Mol. Biol., 215(3), 403-410. https://doi. org/10.1016/S0022-2836(05)80360-2

Ardura, A., Linde, A. R., Moreira, J. C. \& García-Vázquez, E. (2010). DNA bar-coding for conservation and management of Amazonian commercial fish. Biol. Conserv., 143(6), 1438-43. https://doi. org/10.1016/j.biocon.2010.03.019

Baldwin, C. C., Mounts, J. H., Smith, D. G. $\&$ Weigt, L. A. (2009). Genetic identification and color descriptions of early life/history stages of Belizean Phaeoptyx and Astrapogon (Teleostei: Apogonidae) with comments on identification of adult Phaeoptyx. Zootaxa, 2008, 1-22.

Benson, D. A., Cavanaugh, M., Clark, K., Karsch-Mizrachi, I., Lipman, D. J., ... \& Sayers, E. W. (2012). GenBank. Nucleic Acids Res., 41(D1), D36-D42. https://doi.org/10.1093/nar/gks1195

Berry, F. H. \& Barrett, I. (1963). Análisis de las branquiespinas y denominación del arenque de hebra Opisthonema. Inter-A. Tuna Trop. Comm. Bull., 7(2), 110-190. Bingpeng, X., Heshan, L., Zhilan, Z., Chunguang, W., Yanguo, W. \& Jianjun, W. (2018). DNA barcoding for identification of fish species in the Taiwan Strait. PLoS ONE, 13(6), e0198109. https:// doi.org/10.1371/journal.pone.0198109

Bloom, D. D. \& Lovejoy, N. R. (2014). The evolutionary origins of diadromy inferred from a time/calibrated phylogeny for Clupeiformes (herring and allies). Proc. R. Soc. Lond. B. Biol. Sci., 281(1778), 2013-2081. https://doi.org/10.1098/rspb.2013.2081 Bussing, W. \& López, M. (1994). Peces demersales y pelágicos costeros del Pacífico de Centroamérica Meridional. Guía Ilustrada. Rev. Biol. Trop., 47 (Supplemento), 47-164.

Carvalho, D. C., Oliveira, D. A., Pompeu, P. S., Leal, C. G., Oliveira, C. \& Hanner, R. (2011). Deep barcode divergence in Brazilian freshwater fishes: the case of the São Francisco River basin. Mitochondrial DNA, 22(1), 80-86. 
https://doi.org/10.3109/19401736.201 1.588214

Chakraborty, A. \& Iwatsuki. Y. (2006). Genetic variation at the mitochondrial $16 \mathrm{~S}$ rRNA gene among Trichiurus lepturus (Teleostei:Trichiuridae) from various localities: Preliminary evidence of a new species from West Coast of Africa. Hydrobiologia, 563, 501-513. https:// doi.org/10.1007/s10750-006-0105-4

Darriba, D., Taboada, G. L., Doallo, R. \& Posada, D. (2012). jModelTest 2: more models, new heuristics, and parallel computing. Nat. Methods, 9(8), 772. https://doi.org/10.1038/nmeth.2109

Durand, J. D., Hubert, N., Shen, K. N. \& Borsa, P. (2017). DNA barcoding grey mullets. Rev. Fish. Biol. Fish., 27, 233-43. https://doi.org/10.1007/ s11160-016-9457-7

FAO. Organización de las Naciones Unidas para la Alimentación y la Agricultura. (2018). El estado mundial de la pesca y la acuicultura 2018. Cumplir los objetivos de desarrollo sostenible. Italia: FAO.

Fischer, W., Krupp, F., Schneider, W., Sommer, C., Carpenter, K. E. \& Miem, V. H. (1995). Guía FAO para la identificación de especies para los fines de la pesca. Pacífico Centro Oriental. Italia: FAO.

Froese, R. \& Pauly, D. (2019). FishBase. World Wide Web electronic publication. https://www.fishbase.se/search.php

Goodbody-Gringley, G., Strand, E. \& Pitt, J. M. (2019). Molecular characterization of nearshore baitfish populations in Bermuda to inform management. PeerJ, 7, e7244. https://peerj.com/articles/7244/

Hebert, P. D., Ratnasingham, S. \& de Waard, J. R. (2003). Barcoding animal life: cytochrome c oxidase subunit 1 divergences among closely related species. Proc. R. Soc. Lond. B. Biol. Sci., 270
Suppl 1(Suppl 1), S96-S99. https://doi. org/10.1098/rsbl.2003.0025

Huelsenbeck, J. P. \& Ronquist, F. (2001). MRBAYES: Bayesian inference of phylogeny. Bioinf., 17(8), 754-755. https://doi. org/10.1093/bioinformatics/17.8.754

Ivanova, N. V., deWaard, J. R. \& Hebert, P. D. N. (2006). An inexpensive automatization friendly protocol for recovering high quality DNA. Mol. Ecol. Notes, 6(4), 998-1002. https://doi. org/10.1111/j.1471-8286.2006.01428.x

Jahan, H., Akter, M., Begum, R. A. \& R. Shahijahan. (2017). Identification and comparison of three carp fishes based on mitochondrial 16S rRNA gene. $J$. Biol. Sci., 26 (2): 167-174.

Katoh, K., Asimenos, G. \& Toh, H. (2009). Multiple alignment of DNA sequences with MAFFT. In D. Posada (Ed.), Bioinformatics for DNA Sequence Analysis. Methods in Molecular Biology (Methods and Protocols) (pp. 39-64). EE. UU.: Humana Press. https://doi. org/10.1007/978-1-59745-251-9_3

Kocher, T. D., Thomas, W. K., Meyer, A., Edwards, S. V., Paabo, S., ... \& Wilson, A. C. (1989). Dynamics of mitochondrial DNA evolution in animals: amplification and sequencing with conserved primers. Proc. Nat. Acad. Sci. U. S. A., 86(16), 6196-6200. https:// doi.org/10.1073/pnas.86.16.6196

Kochzius, M., Seidel, C., Antoniou, A., Kumar, S., Campo, D., Cariani, A., ... \& Blohm, D. (2010). Identifying fishes through DNA barcodes and microarrays. Plos One, 5(9), e12620. https://doi.org/10.1371/journal. pone. 0012620

Lagúnez, L. \& Rodríguez, F. (1992). Contribución al conocimiento genético de Opisthonema spp. del noreste de México. Inv. Mar. CICIMAR, 7(1),15-24.

Li, C. \& Ortí, G. (2007). Molecular phylogeny of Clupeiformes (Actinopterygii) 
inferred from nuclear and mitochondrial DNA sequences. Mol. Phylogenet. Evol., 44, 386-398. https://doi. org/10.1016/j.ympev.2006.10.030

Meyer, A., Kocher, T. D., BasasibwakI, P. \& Wilson, A. C. (1990). Monophyletic origin of Lake Victoria cichlid fishes suggested by mitochondrial DNA sequences. Nature, 347(6293), 550-553. https://doi.org/10.1038/347550a0

Murase, A., Angulo, A., Miyazaki, Y., Bussing, W. \& López, M. (2014). Marine and estuarine fish diversity in the inner Gulf of Nicoya, Pacific coast of Costa Rica, Central America. Check List, 10(6), 1401-1413. https://www. biotaxa.org/cl/article/view/10.6.1401, https://doi.org/10.15560/10.6.1401

Paine, M., McDowell, J. \& Graves, J. (2007). Specific identification of Western Atlantic Ocean scombrids using mitochondrial DNA cytochrome $c$ oxidase subunit I (COI) gene region sequences. Bull. Mar. Sci., 80(2), 353-367.

Palumbi, S. R. (1996). Nucleic acids II: the polymerase chain reaction. In D. $\mathrm{M}$. Hillis, C. Moritz \& B. K. Mable (Eds.), Molecular systematics (pp. 205-247). EE. UU.: Sinauer \& Associates, Inc.

Peng, Z., He, S. \& Zhang, Y. (2004). Phylogenetic relationships of glyptosternoid fishes (Siluriformes: Sisoridae) inferred from mitochondrial cytochrome b gene sequences. Mol. Phylogenet. Evol., 31(3), 979-987. https://doi.org/10.1016/j.ympev.2003.10.023

Pereira, L. H. G., Hanner, R., Foresti, F. \& Oliveira, C. (2013). Can DNA barcoding accurately discriminate megadiverse Neotropical freshwater fish fauna? BMC Genet., 14, 20. https://doi. org/10.1186/1471-2156-14-20

Pérez-Enríquez, R., Díaz-Viloria, N., Cruz-Hernández, P., Aranceta-Garza, F., Gutiérrez-González, J. L., ... \& Max-Aguilar, A. (2016). Estudios de genética en poblaciones de abulón y sus aplicaciones en ordenamiento pesquero. Rec. Nat. Soc., 2(2), 24-39. https://doi.org/10.18846/ renaysoc.2016.02.02.02.0002

Pérez-Quiñonez, C. I. (2014). Discriminación de las especies del género Opisthonema Gill, 1861 en el Sur del Golfo de California usando análisis morfométricos y genéticos. (Tesis de Maestría no publicada). Centro Interdisciplinario de Ciencias Marinas (CICIMAR), Instituto Politécnico Nacional, México.

Pérez-Quiñonez, C. I., Quiñonez-Velázquez, C. \& García-Rodríguez, F. J. (2020). A simple method for the genetic identification of commercially important species in the Opisthonema genus Gill, 1861 in the southern Gulf of California. Ciencias Marinas, 46(3), 145-154. https://doi.org/10.7773/cm.v46i3.3059

Pérez-Quiñonez, C. I., Quiñonez-Velázquez, C. \& García-Rodríguez, F. J. (2018). Detecting Opisthonema libertate (Günther, 1867) phenotypic stocks in northwestern coast of Mexico using geometric morphometrics based on body and otolith shape. Lat. Am. J. Aquat. Res., 46(4), 779-790.

Pérez-Quiñonez, C. I., Quiñonez-Velázquez, C. \& García-Rodríguez, F. J. (2019). Genetic homogeneity of the Pacific thread herring (Opisthonema libertate) (Günther, 1867) in the Eastern Pacific, inferred from mtDNA sequences. Mitochondrial DNA Part A, 30(3), 517-524. https://doi.org/10.1080/24701394.201 9.1570173

Pérez-Quiñónez, C. I., Quiñónez-Velázquez, C., Ramírez-Pérez, J. S, Vergara-Solana, F. J. \& García-Rodríguez, F. J. (2017). Combining geometric morphometrics and genetic analysis to identify species of Opisthonema Gill, 1861 in the eastern Mexican 
Pacific. J. Appl. Ichthyol., 33(1), 84-92. https://doi.org/10.1111/jai.13051

QGIS. Development Team. (2018). QGIS Geographic Information System. EE. UU: Open Source Geospatial Foundation Project. http://qgis.osgeo.org

Rambaut, A. (2009). FigTree v1.4 2012-2014: Tree Figure Drawing Tool. Edinburgh. Scotland: Institute of Evolutionary Biology, University of Edinburgh. http:// tree.bio.ed.ac.uk/software/figtree

Robertson, D. R. \& Allen, G. R. (2002). Shore fishes of the Tropical Eastern Pacific: an Information System. Smithsonian Tropical Research Institute. http://www.neotropicalfishes.org/sftep

Rocha, A., Garber, N., Garber, A. \& Stuck, K. (2005). Structure of the mitochondrial control region and flanking tRNA genes of Mugil cephalus. Hidrobiológica, 15(2), 139-149.

Rodríguez, J. A. \& Gómez, K. R. (1998). Aspectos relevantes en la biología de Cetengraulis mysticetus (Günther) (Pisces: Engraulidae) en el Golfo de Nicoya, Costa Rica. Uniciencia, 15(1), 57-60.

Rodríguez-Sánchez, R. (octubre, 1985). Aspectos de dinámica poblacional en apoyo de la separación de las especies del género Opisthonema por el método de índice de densidad de branquiespinas. CalCOFI abstracts, annual, conference. EE. UU.

Soto, R. \& Rodríguez, J. (1999). Dinámica poblacional de Opisthonema medirastre (Pisces: Clupeidae) en la costa Pacífica de Costa Rica. Uniciencia, 15(16), 61-64.

Stamatakis, A., Ludwig, T. \& Meier, H. (2005). RAxML/III: a fast program formaximum likelihood/ based inference of large phylogenetic trees. Bioinf., 21(4), 456-463. https://doi.org/10.1093/bioinformatics/ bti191
Teletchea, F. (2009). Molecular identification methods of fish species: reassessment and possible applications. Rev. Fish. Biol. Fish., 19(3), 265-293. https://doi. org/10.1007/s11160-009-9107-4

Vega-Corrales, L. A. (2010). Evaluación poblacional del stock explotable del complejo Opisthonema (Pisces: $\mathrm{Cu}$ pleidae) en el Golfo de Nicoya, Costa Rica. Rev. Cien. Mar. Cos., 2, 83-94. https://doi.org/10.15359/revmar.2.7

Vicente, F., Loeb, M. V., Paiva, A. C. G. D., Sampaio, C. L., Argolo, L. A. \& Jacobina, U. P. (2020). Integrative systematics unveils the controversial identity of Engraulidae fishing stocks in a Neotropical estuary, northeast Brazil. Neotrop. Ichthyol., 18(4), 1-17. https://doi. org/10.1590/1982-0224-2020-0037

Wang, P., Zhao, C., Fan, S., Yan, L. \& Qiu, L. (2018). The complete mitochondrial genome of Thryssa hamiltonii and phylogenetic analysis of Engraulidae (Clupeiformes; Clupeoidei). Mitochondrial DNA Part B., 3(2), 538-540. https://doi.org/10.1080/23802359.2018. 1467232

Ward, R. D., Zemlak, T. S., Innes, B. H., Last, P. R. \& Hebert, P. D. N. (2005). DNA barcoding Australia's fish species. Phil. Trans. R. Soc., 360(1462), 1847-1858. https://doi.org/10.1098/rstb.2005.1716

Wortley, H. \& Scotland, R. (2006). The effect of combining molecular and morphological data in Published Phylogenetic Analyses. Syst. Biol., 55(4), 677-685. https://doi. org/10.1080/10635150600899798 